\title{
A DEATH DUE TO ACUTE AORTIC DISSECTION
}

\section{Rathnaweera RHAI}

DOI :

http://doi.org/10.4038/sljfmsl. v11i2.7859

Corresponding Author Rathnaweera RHAI Department of Forensic Medicine, Faculty of Medicine, Karapitiya, Galle

ajithrathnaweera@gmail.com https://orcid.org/00000002-7418-2981

Key Words

Acute aortic syndrome, aortic dissection, haemopericardium

Article History

Received: 05.04.2020

Received in revised form: 06.07.2020

Accepted: 20.07.2020

Available online: 21.12.2020

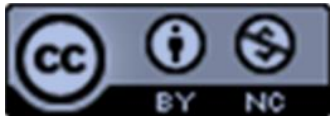

This article is licensed under the terms of the Creative Commons Attribution-Non Commercial 4.0 International License.

e-ISSN: 2465-6089

\section{ABSTRACT}

Acute aortic syndrome (AAS) is a term used to describe a collection of life-threatening aortic disease conditions that have similar presentations, but differ in their demographic, pathological, clinical and other characteristics. The exact number of deaths due to AAS is unknown. However studies suggest that the majority of deaths in AAS are due to acute aortic dissection. A 51-year-old male developed sudden onset chest pain while he was taking a bath in the evening. It was a sharp, tearing type chest pain, which was radiating to the left side of his neck. On admission to hospital his low blood pressure was found to be low. He suffered cardiac arrest a few minutes later and died without regaining consciousness. Post-mortem examination revealed a massive haemopericardium $(600 \mathrm{ml})$. Aortic dissection was identified extending from the aortic root to the lower part of the descending abdominal aorta. The thickest point of the dissection was 1.5 $\mathrm{cm}$. There was an intimal tear inferiorly. The dissection extended through the aortic arch to the descending aorta ending 1 $\mathrm{cm}$ above the renal artery branching point. Sections of the aorta showed cystic medial degeneration with prominent pools of mucin. Mild atheroma was seen in some sections. The presence of aortic dissection was confirmed. The cause of death was given as haemopericardium due to ruptured aortic dissection.
Acute aortic dissection may mimic other cardiovascular conditions, especially ischemic cardiac events. Therefore, the clinical suspicion and a comprehensive historytaking are the key for an early diagnosis. Careful dissection of the aorta and its branches is essential when making a postmortem diagnosis of aortic dissection. Proper histopathology examination of the aorta assisted immensely in confirming the diagnosis.

\section{INTRODUCTION}

Acute aortic syndrome (AAS) is a term used to describe a collection of life-threatening aortic disease conditions that have similar presentations, but differ in their demographic, pathological, clinical and other characteristics. ${ }^{1}$ Diseases of aorta and its branches accounts for approximately 43,000 to 47,000 deaths annually in the United States alone. ${ }^{2}$ The three major diseases that comprise the AAS includes aortic dissection (AD), intramural haematoma (IMH) and penetrating aortic ulcer $(\mathrm{PAU}){ }^{2}$ The differentiation between these conditions is difficult as all of them present with acute onset severe chest or back pain with similar radiological findings. ${ }^{2}$ The exact number of deaths due to AAS is unknown. However autopsy studies suggest that the majority of deaths in AAS are due to acute aortic dissection. ${ }^{2}$ The annual incidence of aortic dissections in the United States is around 3-4 cases per $100000 .^{3}$ 
Preadmission mortality is reported to be around $20 \%$ whereas it is around $30 \%$ in hospital admissions. ${ }^{3,4}$ The annual incidence is reported to be 6 cases per 100,000 in United Kingdom whereas it ranged from 9.1 per 100,000 in women to 16.3 per 100,000 in men in Sweden. ${ }^{5}$

There are two leading theories regarding the development of aortic dissection. ${ }^{6}$ In the first, the development of an intimal tear is believed to be the triggering event, which is followed by seepage of blood from the aortic lumen to the already weakened medial space due to elastic degeneration and smooth muscle cell loss. In the second theory rupture of the vasa vasorum is the initial event, which leads to bleeding within the aortic wall which subsequently leads to intimal disruptions and propagation of a dissection flap.

The propagation of the aortic dissection usually happens in an ante grade manner due to the pressure wave exerted through the left ventricle, though the retro grade propagation is also a possibility. ${ }^{6}$ The expansion of the false lumen can lead to compression of the true lumen with resultant under perfusion of vital organs. The ultimate life-threatening complications could include pericardial effusion causing cardiac tamponade, exsanguination from aortic rupture, cardiac ischaemia and myocardial infarction, cerebrovascular accidents, mesenteric ischaemia, ischaemic colitis and spinal ischaemia. ${ }^{6}$

\section{CASE REPORT}

A 51-year-old male, who was a heavy smoker and an alcoholic, without any significant past medical history, developed sudden onset chest pain while he was taking a bath in the evening. It was a sharp, tearing type chest pain, which radiated to the left side of his neck. He had returned home after a hard day's work and had consumed alcohol on his way home. He has not had such chest pain in the past. Later he complained of severe back ache and difficulty in breathing. He was taken to the closest hospital by his family members, from where he was transferred to a Teaching hospital. He was found to have a low blood pressure on admission. He went into cardiac arrest few minutes later. Cardio-pulmonary resuscitation was provided for 30 minutes without any success. An inquest was requested by the attending doctors and the Inquirer into Sudden death ordered a post-mortem examination.

The body was that of an adult male with features in keeping with the stated age, weighing 66 kilograms and measuring approximately $168 \mathrm{~cm}$ in height with a body mass index of $23.5 \mathrm{~kg} / \mathrm{m}^{2}$. No injuries were identified. There were no discharges present at ear, nose or throat.

The heart weighed $390 \mathrm{~g}$. The pericardial cavity contained approximately $600 \mathrm{ml}$ of predominantly liquid and some clotted blood. Epicardium was unremarkable. No acute ischaemic changes were seen in the myocardium. The right ventricular wall thickness was $4 \mathrm{~mm}$. The left ventricular free wall thickness was $14 \mathrm{~mm}$. The interventricular septum measured $13 \mathrm{~mm}$ in thickness. The endocardium appeared unremarkable. The cardiac valves were normal in configuration. The coronary arteries were normal in configuration with a right dominant circulation. The right and left coronary ostia were patent. There was mild calcific atherosclerosis of all three vessels without significant stenosis. There was an aortic dissection extending from the aortic root (Fig. 1) to the lower part of the descending abdominal aorta (Fig. 2). The thickest point of the dissection was $1.5 \mathrm{~cm}$. There was an intimal tear inferiorly. The dissection extended through the aortic arch to the descending aorta ending $1 \mathrm{~cm}$ above the renal artery branching point. The renal arteries and distal branches were not involved. Fatty streaks and atherosclerotic changes were noted in the aortic arch and the descending aorta. 


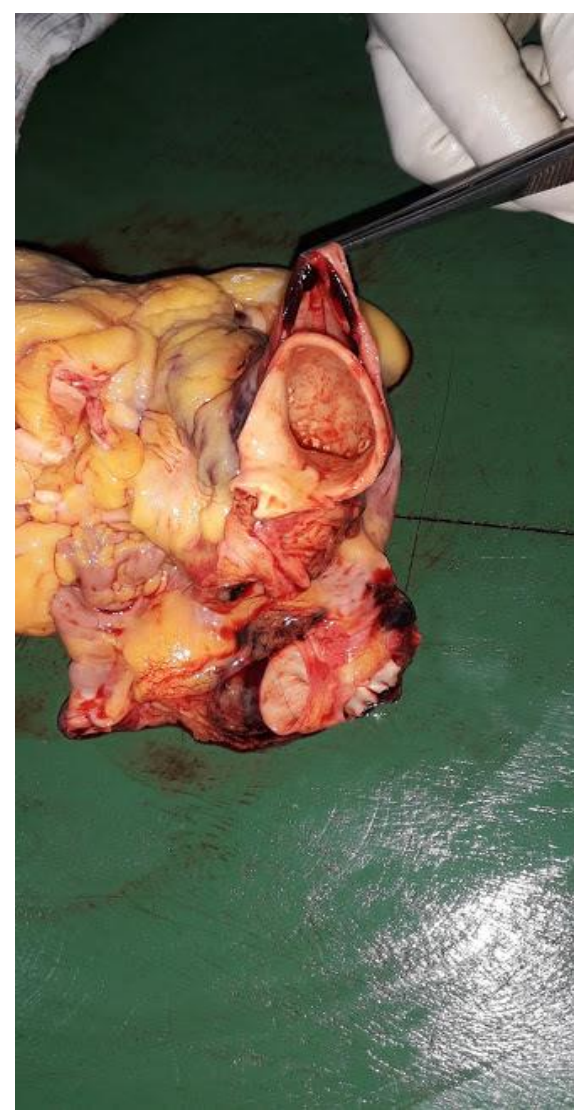

Fig. 1: Aortic dissection involving the root of aorta

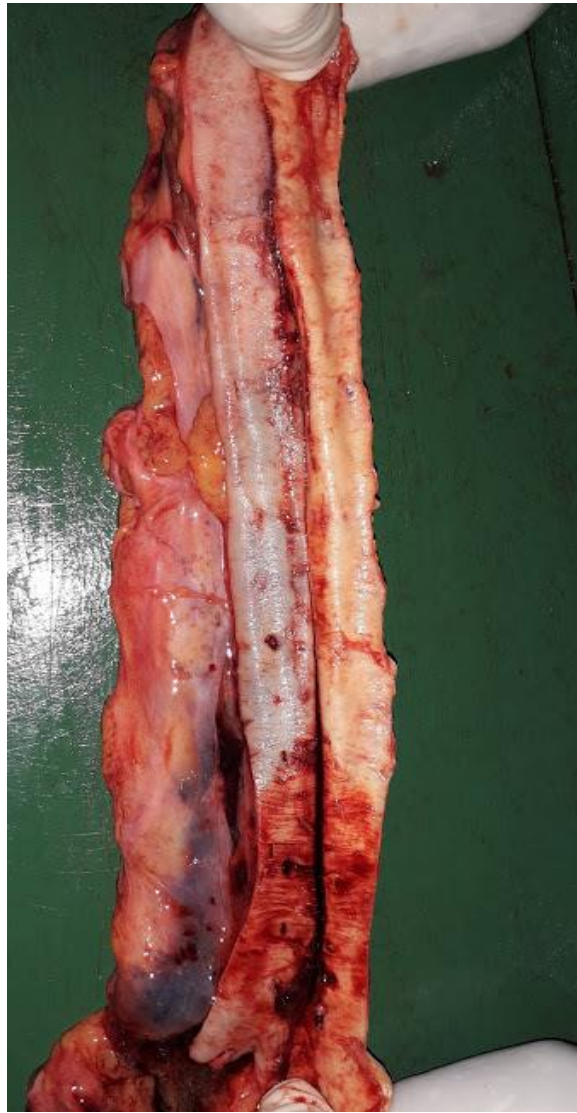

Fig.2: Aortic dissection extending in to the descending aorta
Both pleural cavities were free from plaques. The visceral pleurae were smooth. The left lung weighed $490 \mathrm{~g}$ and the right lung weighed 482 g. Both lungs appeared well inflated. There was no consolidation or collapse. No focal mass lesions were identified. There was no thrombosis or infarction. The brain was normal in configuration. No appreciable gyral atrophy was seen. Serial coronal sections through the cerebral hemispheres revealed no abnormality of the cerebral cortex, underlying white matter or basal ganglia. The stomach contained semi digested food. The liver weighed $1710 \mathrm{~g}$. The capsule was intact and the parenchyma was fatty with early signs of cirrhosis. The left kidney weighed $130 \mathrm{~g}$ and the right $145 \mathrm{~g}$. On cut section the cortices were of normal thickness and the cortico-medullary junction was well defined. The renal papillae and renal pelvis were unremarkable. The ureters were unobstructed. There was no obvious lymphadenopathy. The spleen weighed $165 \mathrm{~g}$ and was unremarkable.
Histopathology of all organs were performed. The myocardium showed mild perivascular and interstitial fibrosis with some associated cardiomyocyte hypertrophy. There were no acute ischaemic changes. Coronary Arteries showed up to $30 \%$ stenosis. There was no luminal thrombus. Sections of the aorta showed cystic medial degeneration with prominent pools of mucin. Mild atheroma was seen in some sections. The presence of aortic dissection was confirmed (Fig. 3, Fig 4).

The lungs showed moderate amount of pulmonary oedema with intra-alveolar, haemosiderin laden macro phages. The liver showed largely preserved lobular architecture with macrovescicular fatty change and early bridging fibrosis. No interface or lobular hepatitis was present. The kidneys showed rare globally sclerosed glomeruli and occasional periglomerular fibrosis. The arteries showed minimal intimal thickening. There was no significant inflammation. 


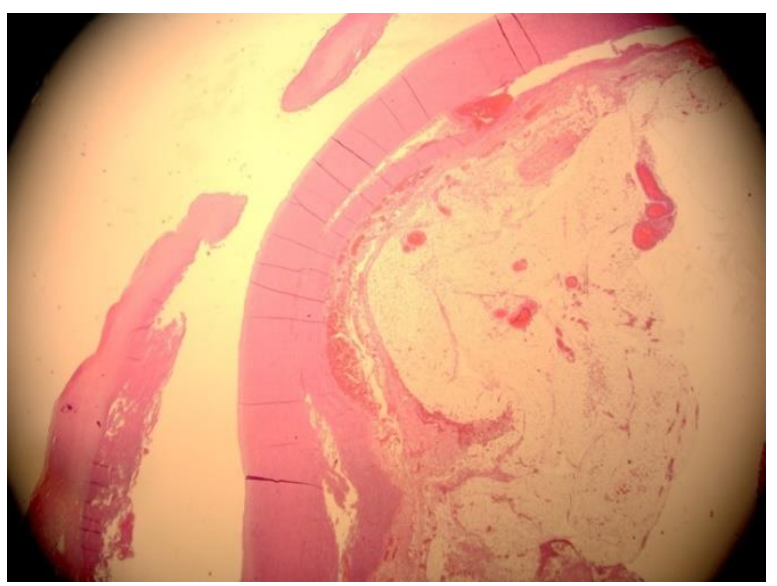

Fig. 3: Histopathology of the aortic dissection ( $H$ \& $E$ stain - low power) - note the defect in tunica media, cystic medial degeneration with disruption of the elastic lamina and abundant fresh blood in the adventitia

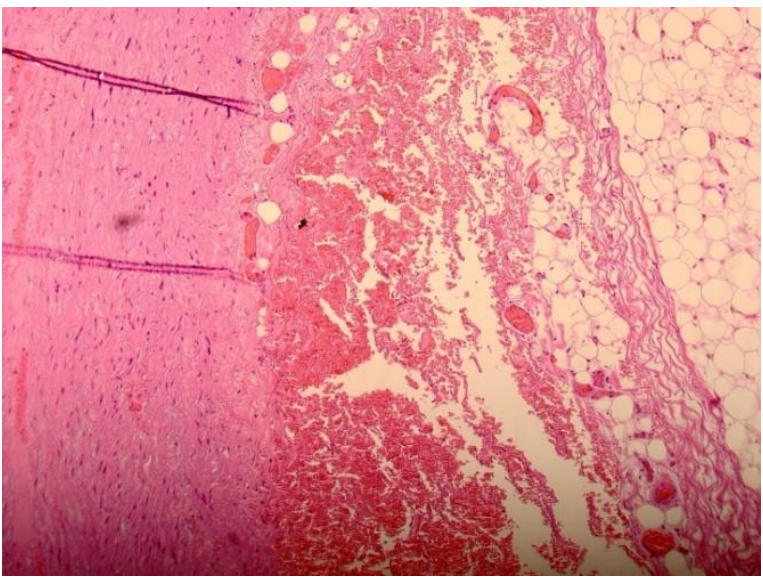

Fig. 4: Histopathology of the aortic dissection ( $H$ \& E stain - high power) - note the defect in tunica media, cystic medial degeneration with disruption of the elastic lamina and abundant fresh blood in the adventitia

The cause of death was given as haemopericardium due to ruptured aortic dissection.

\section{DISCUSSION}

Aortic dissection is relatively uncommon but is a devastating condition, because once it occurs, it may lead to acute haemodynamic compromise. The location of the entry tear plays a key role in treatment and outcome. ${ }^{3}$ The two commonly used classifications in aortic dissections are Stanford classification and DeBakey classification. ${ }^{3,5,6}$ Both these classifications are based on anatomical location and the extent of the dissection flap. In Stanford classification, type A comprises of all dissections that affect the ascending aorta, whereas type $B$ includes all dissections that do not affect the ascending aorta (begins distal to the origin of the left subclavian artery, involving the descending aorta and/or abdominal aorta, without affecting the ascending aorta). According to DeBakey classification, type I dissection originates in the ascending aorta; propagates at least to the aortic arch and often beyond distally. The type II dissection originates and confined to the ascending aorta alone whereas type III dissections are limited to the descending aorta. These classifications systems allow for a standard language in describing aortic pathology, and also reflect the importance of anatomic location of aortic dissection in dictating treatment and determining prognosis.

Ascending aorta is affected twice as that of descending aorta in acute aortic dissection. Aortic dissection involving ascending aorta is mostly found among patients in the age group of 50 to 60 years whereas aortic dissection involving descending aorta is commonly found in the age group of 60 to 70 years. ${ }^{4,5,6}$ The acute type $A$ dissection which involves the ascending aorta is a surgical emergency. ${ }^{7}$

This carries a mortality rate of $1-2 \%$ per hour within the first day and up to $50 \%$ within the first 48 hours if left untreated. ${ }^{7,8}$ The acute type $B$ dissection is not as life threatening as Type A dissection, however it also has a mortality rate of $10 \%$ within the first month. ${ }^{7}$

In this case, the dissection was found to be involving the ascending aorta, aortic arch and the descending aorta running up to the origin of renal arteries. According to Stanford classification, it is a Type A dissection, whereas according to DeBakey classification, it is a type I dissection. As the literature suggested, the type A dissections present more abruptly with high mortality rate. 
This patient collapsed within 2 hours of development of initial symptoms and did not recover afterwards.

Patients with acute aortic dissection, typically presents with sudden onset, sharp, tearing or stabbing type chest pain. ${ }^{3}$ Some may present with back or abdominal pain as well. ${ }^{9}$ Sudden onset of severe, sharp chest pain is the classic presenting symptom in type $A$ dissection. ${ }^{6}$

Type B dissections are more commonly associated with abdominal pain ${ }^{6}$ and back pain than type A dissection. In type A dissection, this pain usually radiates to the neck whereas in type $B$ dissection, it radiates to the interscapular region. ${ }^{3}$ Pain may be absent in $10 \%$ of the patients. ${ }^{3}$ This asymptomatic presentation is mostly seen among diabetic patients. $^{3}$ The presenting symptom of chest pain can sometimes mimic that of acute coronary syndrome. The possibility of acute aortic dissection extending into the coronary ostia, there by actually causing an acute ischaemic event, further complicates the presentation. Therefore, in every suspected cardiac ischaemic event, acute aortic dissection should be considered as one of the differential diagnosis. ${ }^{9}$ Some patients can present with sudden loss of consciousness if the acute aortic dissection causes a rapid blood loss. ${ }^{3}$ Syncope is another important presenting symptom of acute aortic dissection and it could be due to complications such as cardiac tamponade or carotid dissection. ${ }^{9}$ Dissection related occlusion of aortic branches can lead to ischemia in any adjacent organs. ${ }^{9}$ Interrupted perfusion may result in neurological complications, limb ischemia and visceral ischemia. ${ }^{3}$ From a hemodynamic perspective, the blood pressure can be high or low in different patients with acute aortic dissections. Hypotension is commonly seen in patients with type $A$ dissection whereas in type $B$ dissection, hypertension is the predominant finding. ${ }^{3}$

The presenting complaint in this case was sudden severe chest pain of abrupt onset, which radiated to the left side of neck.
On admission to the hospital, he was found to have a low blood pressure. These presenting features are suggestive of a Type A dissection and the differentiation from an acute coronary event based only from these findings is very difficult.

A variety of risk factors are known among patients with aortic dissection. It can affect men and women of all ages but is most commonly seen in men between 60 and 80 years of age. ${ }^{7}$ Risk factors for aortic dissection can be divided into iatrogenic, acquired and inherited categories. ${ }^{6}$

latrogenic causes accounts for approximately $5 \%$ of aortic dissections. ${ }^{6}$ Percutaneous procedures and cardiac surgery are among the common iatrogenic causes that predispose to aortic dissection. Aortic dissection is estimated to occur in $0.02 \%$ of cardiac catheterization procedures. $^{6}$ A higher incidence $(0.03 \%)$ is reported during percutaneous coronary intervention. Patients undergoing open-heart surgery have an increased risk of developing aortic dissection and these are typically Type A dissections. ${ }^{6}$ In this case, this patient has not undergone any of these procedures.

Among the acquired risk factors, hypertension (present in $75 \%)^{6,10}$ and atherosclerosis (present in $30 \%)^{6,11}$ are commonly seen among patients with aortic dissection. Hypertension is a more common finding in patients with type $B$ dissections (70\%) than in type $A$ dissection $(36 \%){ }^{6,11}$ Hypertension, pregnancy, cocaine and amphetamine abuse, high intensity weight lifting and heavy exercise are all known to cause increase stroke volume and intra-aortic pressure, thereby weakening the arterial wall, predisposing to the development of aortic dissection. ${ }^{6}$ Tobacco use and smoking are known additional risk factors. They can cause medial wall degeneration through breakdown of collagen and elastin, thereby increasing the risk of aortic dissection. ${ }^{6}$ 
In this case, this patient was a heavy smoker. He also was a manual worker who used to lift heavy weights on a regular basis. These factors would have increased the risk of developing aortic dissection.

Certain connective tissue disorders can cause inherent weakening of the aortic wall and subsequent aortic dissection. Syndromes such as Marfan with fibrillin defects, Ehlers- Danlos type IV with abnormal synthesis of type III procollagen, Loeys-Dietz, annulo-aortic ectasia, familial aortic dissection and other connective tissue disorders associated with cystic medial necrosis are among the well-known causes. ${ }^{3,6}$ Aortic dissection is six times more common among patients with Turner syndrome. ${ }^{6}$ Patients with certain congenital cardiovascular abnormalities are known to have a higher risk in developing aortic dissections. Conditions such as bicuspid aortic valves and coarctation of aorta are among the well-known associated causes. $^{3}$

In this case, this patient did not have a history of any of these mentioned disease conditions. Family members were unaware of any of these conditions among any of them. However, a proper screening and genetic counselling among the remaining members are important as these syndromes may go unreported.

\section{CONCLUSIONS}

Acute aortic dissection may mimic other cardiovascular conditions, especially ischemic cardiac events. Therefore, clinical suspicion and comprehensive history-taking are key for an early diagnosis. The importance of having a thorough knowledge on predisposing conditions and characteristic clinical features is highlighted here. Careful dissection of the aorta and its branches is essential when making a post-mortem diagnosis of aortic dissection. Proper histopathological examination of the aorta assisted immensely in confirming the diagnosis.

\section{ETHICAL ISSUES}

None

\section{CONFLICTS OF INTEREST}

There are no conflicts of interest.

\section{AUTHOR CONTRIBUTIONS}

RHAIR: Conception or design of the work, interpretation of data for the work and drafting the work. 


\section{REFERENCES}

1. Vilacosta I, Román JA. Acute aortic syndrome. Heart. 2001; 85(1):365-368. doi: 10.1136/heart.85.4.365

2. Coevera J. Acute aortic syndrome. Annals of Cardiothoracic Surgery. 2016 May; 5(3): 188193. doi: 10.21037/acs.2016.04.05.

3. Thrumurthy SG, Karthikesalingam A, Patterson BO, Holt PJE, Thompson MM. The diagnosis and management of aortic dissection. British Medical Journal. 2011 Jan;344(1):1-7. doi: 10.1136/bmj.d8290.

4. Olsson C, Thelin S, Stahle E. Thoracic aortic aneurysm and dissection: increasing prevalence and improved outcomes reported in a nationwide population-based study of more than 14000 cases from 1987 to 2002. Circulation. 2006; 114(1):2611-2618. doi:10.1161/CIRCULATIONAHA.106.630400

5. Goldfinger JZ, Halperin JL, Marin ML, Stewart AS, Eagle KA, Fuster V. Thoracic Aortic Aneurysm and Dissection. Journal of the American College of Cardiology. 2014;64(16):1725-1739. doi: 10.1016/ j.jacc.2014.08.025
6. Al'Aref JS, Girardi NL, Devereux R, et al. A Contemporary Review of Acute Aortic Dissection. Emergency Medicine (Los Angel). 2015; 5(5):1-10. doi: 10.4172/21657548.1000274

7. Salameh M, Ratchford E. Aortic dissection. Vascular Medicine. 2016; 21(3): $276-278$. doi:10.1177/1358863X16643601

8. Ince $\mathrm{H}$, Nienaber $\mathrm{CA}$. Diagnosis and management of patients with aortic dissection. Heart. 2007; 93(1): 266-270. doi: $10.1136 /$ hrt.2005.078550

9. Silaschi $M$, Byrne J, Wendler O. Heart. 2017;103(1):78-87. .doi: 10.1136/heartjnl2015-308284

10. Januzzi JL, Isselbacher EM, Fattori $R$, et al. Characterizing the young patient with aortic dissection: results from the International Registry of Aortic Dissection (IRAD). Journal of the American College of Cardiology. 2004; 43(1):665-669. doi: 10.1016/j.jacc.2003.08.054

11. Hagan PG, Nienaber CA, Isselbacher EM, et al. The International Registry of Acute Aortic Dissection (IRAD): new insights into an old disease. The Journal of American Medical Association. 2000; 283(1): 897-903. doi:10.1001/jama.283.7.897 